\title{
Cluster Synchronization in Multiplex Networks
}

\author{
Sarika Jalan ${ }^{1,2}$ and Aradhana Singh ${ }^{1}$ \\ ${ }^{1}$ Complex Systems Lab, Discipline of Physics, Indian Institute of Technology Indore, Indore-452017 and \\ ${ }^{2}$ Centre for Bio-Science and Bio-Medical Engineering, \\ Indian Institute of Technology Indore, Indore-452017
}

\begin{abstract}
We study the impact of interaction of nodes in a layer of a multiplex network on the dynamical behavior and cluster synchronization of these nodes in other layers. We find that nodes interactions in one layer affects the cluster synchronizability of another layer in many different ways. While multiplexing with a sparse network enhances the synchronizability multiplexing with a dense network suppresses the cluster synchronizability with the network architecture deciding the impact of the enhancement and suppression. Additionally, at weak couplings the enhancement in the cluster synchronizability due to multiplexing remains of the driven type, while for strong couplings the multiplexing may lead to a transition to the self-organized mechanism.

PACS numbers: 05.45.Xt,05.45.Pq
\end{abstract}

Synchronization is an universal phenomenon observed in a range of the systems [1]. It is known to be important for proper functioning of many complex systems. For example in brain, the phase synchronization at different locations is responsible for action, motion, vision and sensation [3]. Recently, cluster synchronization has gained tremendous attention due to its occurrence and importance in real world systems [4 6 ]. The cluster synchronization refers to the case when the nodes of a system divide into several synchronized groups so that the nodes in the same group synchronize with each other while do not synchronize with the nodes in the different groups. So far the studies on the cluster synchronization have mainly focused on complex systems being represented as isolated networks [ 11], however a complex system may consist of a superposition of a number of interacting networks 12 14], such as a social system which is composed of different sub-networks consisting family, friends, colleagues, work collaborators and hence forming a multiplex network. The multiplex network presents a more realistic representation of real world interactions [12] leading to a spurt in the activities of modeling real world complex systems. Most of these studies have concentrated on the in- vestigation of various structural properties or emergence of spectral properties [15 17]. Few works considering dynamical properties of the multiplex networks report that the synchronizability of a multiplex network is maximum for the small-world - random regular topology [18] and multiplexing reduces the rate of the global synchronization [18].

In this Rapid communication, we study dynamical behavior of nodes in a layer upon multiplexing with another layer. Particularly, we investigate the impact of nodes interactions in one layer on the cluster synchronization of the same nodes in the other layer. In a realistic situation, the connection density as well as degree distribution of two layers can be different, for instance in a social system a family network can be denser than a counter friendship network. Similarly, the friendship network can be denser than a corresponding business network. In the present paper, we explore the impact of the network architecture on the cluster synchronizability of another layer. We find that nodes interacting in one layer affect the synchronizability of another layer in many different ways. While multiplexing with sparse networks enhances the cluster synchronizability of a layer, impact of multiplex- 
ing with dense networks depend on the network architecture. The enhancement in cluster synchronization is referred to when number of the nodes forming synchronized clusters increases. Furthermore, we report that the change in the density of connections in a layer of the multiplex network may bring a change in the mechanism of the cluster synchronization in another layer. Previous studies on dynamical behavior of isolated networks have identified two different mechanisms of cluster synchronization namely, the driven (D) and the self-organized (SO) 5]. The D and SO synchronization correspond to the synchronization due to the inter and intra cluster couplings, respectively.

We consider the well known coupled maps model [19] to investigate the phase synchronized clusters in the multiplex networks. We consider the phase synchronization instead of the complete synchronization as for sparse networks number of nodes exhibiting the complete synchronization is very less and with an increase in the connection density there is a transition to the globally synchronized state [12], whereas the prime motive of the current work is to study cluster synchronization. The phase synchronization reveals interesting cluster patterns as well as dependence of mechanism of cluster formation in one layer on the network structure of another layer. Let each node of the network be assigned a dynamical variable $x^{i}, i=1,2, \ldots, N$. The dynamical evolution is defined

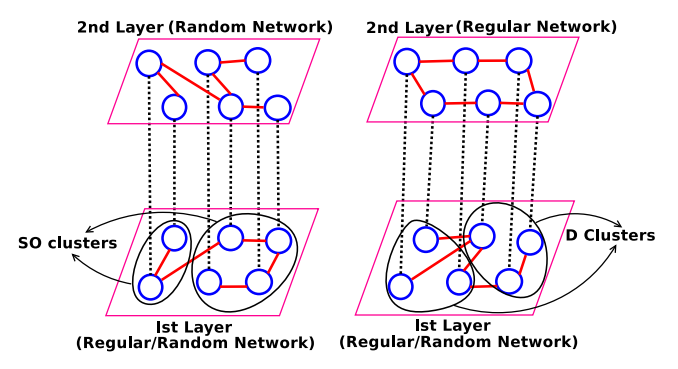

FIG. 1: Schematic diagram depicting a multiplex network with two layers. The dashed lines indicate the inter-layer connections. The density of connections in the different layers can be different and is defined as $\left\langle k_{1}\right\rangle$ for the first layer and $\left\langle k_{2}\right\rangle$ for the second layer. by,

$$
x_{i}(t+1)=(1-\varepsilon) f\left(x_{i}(t)\right)+\frac{\varepsilon}{k_{i}} \sum_{j=1}^{N} A_{i j} g\left(x_{j}(t)\right)
$$

Here, $A$ is the adjacency matrix with elements $A_{i j}$ taking values 1 and 0 depending upon whether there is a connection between $i$ and $j$ or not. Degree of a node is given as, $k_{i}=\sum_{j=1}^{N} A_{i j}$ and $\varepsilon$ is the overall coupling constant and $N$ is total number of nodes in a layer. The average degree of the different layers may be different and are indicated as $\left\langle k_{1}\right\rangle$ and $\left\langle k_{2}\right\rangle$. The function $f(x)$ defines a local nonlinear map, whereas $g(x)$ defines the nature of coupling between the nodes. We consider phase synchronization defined as follows [5]. Let $n_{i}$ and $n_{j}$ denote the number of times when the variables $x_{i}(t)$ and $x_{j}(t)$, $t=1,2, \ldots T$ for the nodes $i$ and $j$ exhibit local minima during the time interval $T$. Let $n_{i j}$ denotes the number of times these local minima match with each other. The phase distance between two nodes $i$ and $j$ is then given as $d_{i j}=1-2 n_{i j} /\left(n_{i}+n_{j}\right)$. The nodes $i$ and $j$ are phase synchronized if $d_{i j}=0$. All the pairs of nodes in a cluster are phase synchronized [23].

We evolve Eq. 1 starting from a set of random initial conditions and study the phase synchronized clusters after an initial transient. We present detailed results of cluster synchronization for simplest multiplex network consisting of two layers. First layer can be represented by a regular or a random network, similarly the second layer can also be modeled by a regular or random network. Here, we present results for all the possible combinations, such as random-random, random-regular, regular-regular and regular-random. First, we discuss the cluster synchronizability of a regular network represented by 1-d lattice upon multiplexing with a ER random network [14]. We find that the isolated sparse 1-d lattice at weak couplings leads to the phase synchronized clusters with all the nodes participating in the clusters, whereas strong couplings lead to a very few nodes forming clusters (Fig. 2(a)). Multiplexing with a sparse ER network enhances the cluster synchronizability of the 1-d lattice 


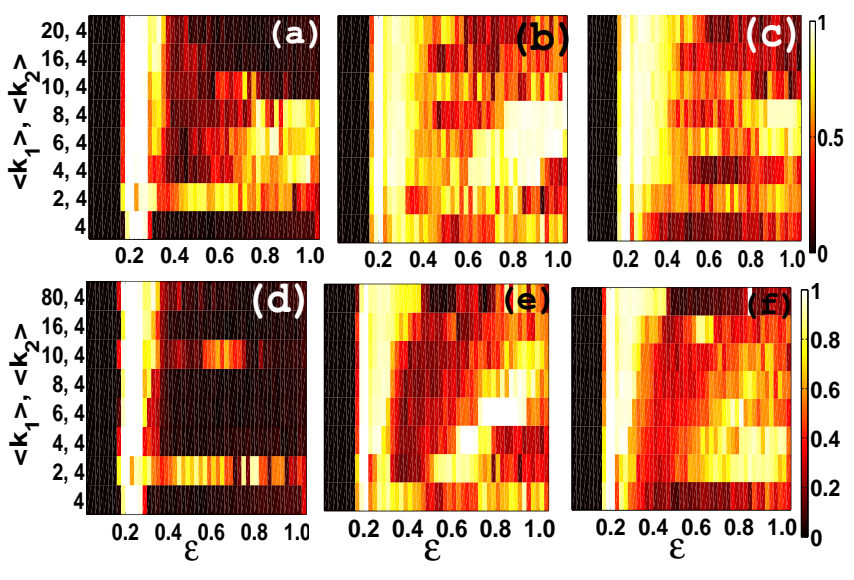

FIG. 2: Phase diagram depicting the variation of $f_{\text {clus }}$ with respect to the $\varepsilon$ and the average degree $\left(\left\langle k_{2}\right\rangle\right)$ of a layer with 1-d lattice (top panel) and random network (bottom panel) architecture for (a) 1-d lattice, (b) SF networks and (c) random networks. The average degree of the second layer $\left(\left\langle k_{1}\right\rangle\right)$ remains same for all three cases. For all the layers $N=100$ and phase diagrams are plotted for average over 20 random realizations of the networks and initial conditions.

at all the couplings. Multiplexing with a denser ER network while enhances the cluster synchronizability as weak couplings, leaves the cluster synchronizability unchanged with few nodes keep forming synchronized clusters at the intermediate and strong couplings. Fig. 2(a) demonstrates that cluster synchronizability of 1-d lattice enhances at the weak couplings irrespective of the value of $\left\langle k_{2}\right\rangle$, whereas at the intermediate coupling, for $\left\langle k_{2}\right\rangle=2$, there is an enhancement in the cluster synchronization, which for the higher values of $\left\langle k_{2}\right\rangle$ gets vanished. At strong couplings synchronization enhances for $\left\langle k_{2}\right\rangle \lesssim 8$. Additionally, the multiplex network yields the chaotic dynamics for almost all the coupling values for the layers being represented by sparse networks. Note that the synchronizability of the second layer, represented as the ER random network, always increases with an increase in the average degree as observed for the isolated networks.

Next we discuss the cluster synchronizability of the 1d lattice upon multiplexing with various other network architectures. At the weak couplings, multiplexing with

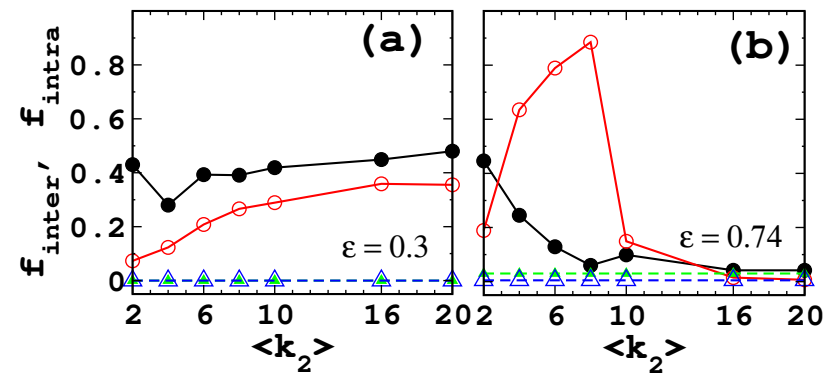

FIG. 3: Variation of $f_{\text {inter }}$ and $f_{\text {intra }}$ with $\left\langle k_{2}\right\rangle$ for isolated 1-d lattice (close and open triangles) with $N=100$ and $\left\langle k_{1}\right\rangle=4$ and after multiplexing with a random network (close and open circles) with various average degrees $\left(\left\langle k_{2}\right\rangle\right)$. All the graphs are plotted for an average over twenty realizations of the initial conditions. Value of $\varepsilon$ are chosen such that they exhibit an enhancement in the D synchronization and enhancement in the SO synchronization followed by a suppression at the strong couplings with an increase in $\left\langle k_{2}\right\rangle$. All the graphs are plotted for average over 20 different realizations of network and initial conditions.

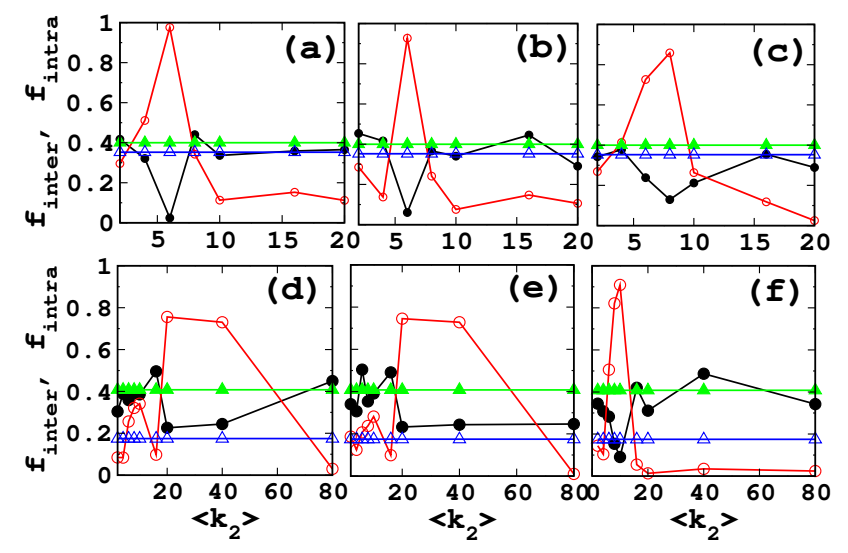

FIG. 4: Variation of $f_{\text {inter }}$ and $f_{\text {intra }}$ with $\left\langle k_{2}\right\rangle$ for isolated SF network (close and open triangles) and for SF network after multiplexing (closed and open circles) with (a) 1-d lattice, (b) SF networks, and (c) random networks at $\varepsilon=0.74$ (top panel) and $\varepsilon=1.0$ (bottom panel). All the graphs are plotted for average over 20 different realizations of network and initial conditions.

the 1-d lattice and SF networks lead to an enhancement in the cluster synchronizability as observed for the multiplexing with the ER random network. At the strong couplings, there is an enhancement in the synchroniza- 


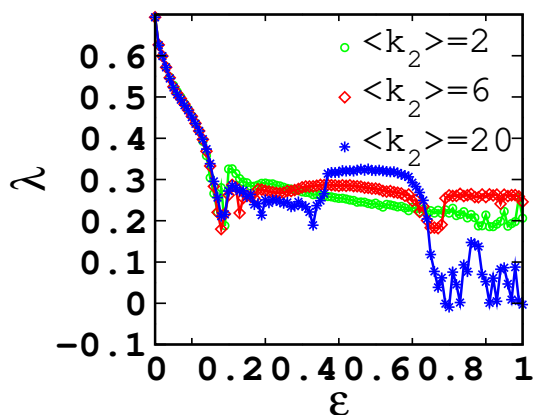

FIG. 5: The largest Lyapunov exponent for a multiplex network consisting of two layers, one represented with the ER random $\left(\left\langle k_{1}\right\rangle=4\right)$ network and another with 1-d lattice for various average degree $\left\langle k_{2}\right\rangle$. Number of nodes in each layer is $N=100$.

tion for sparse networks as observed for the multiplexing with the random networks but the connection density for which this enhancement occurs becomes lower. For example, a 1-d lattice with $\left\langle k_{1}\right\rangle=4$, exhibits no enhancement in the cluster synchronization upon multiplexing with the 1-d lattice and SF networks with $\left\langle k_{2}\right\rangle \gtrsim 4$ and $\left\langle k_{2}\right\rangle \gtrsim 6$ respectively (Fig. 2(a)). Thus the enhancement in the cluster synchronizability of the 1-d lattice is least favorable when it is multiplexed with the 1-d lattice and favorable being multiplexed with the random networks.

Further, we study cluster synchronizability of SF networks upon multiplexing with various network architecture. The isolated sparse SF networks are known to exhibit a better cluster synchronizability as compared to the sparse regular or random networks (Fig. 2(b)). At the weak couplings, the multiplexing with another network only changes the cluster pattern and does not bring any enhancement in the cluster synchronization. For example the isolated SF networks with $\left\langle k_{1}\right\rangle=4$ and $N=100$ lead to the participation of about $50 \%$ nodes in the cluster formation. After multiplexing, the same fraction of the nodes keep participating in the cluster formation as shown by the reappearance of the grey shade in the Fig. 2(b). At the intermediate and strong couplings there is an enhancement in the synchronization for multiplexing with the sparse networks whereas impact of multiplexing with the dense networks largely depend on the network architecture. For example, the cluster synchronizability of SF networks with $\left\langle k_{1}\right\rangle=4$ enhances for $\left\langle k_{2}\right\rangle \lesssim 40$ (Fig. 4(e) and (f)) upon the multiplexing with 1-d lattice and SF networks. For the higher connection density there is a suppression in the synchronization, while in the case of multiplexing with the random network the enhancement occurs for $\left\langle k_{2}\right\rangle \lesssim 12$ (Fig. $4(\mathrm{f})$ ) and with a further increase in the connection density cluster synchronization suppresses. This shows that the cluster synchronizability of the SF network is favorable when it is multiplexed with the 1-d lattice and SF networks. Furthermore, multiplexing of ER random networks with different network architectures at the weak couplings exhibit the similar behavior as observed for the 1-d lattice, whereas the strong couplings lead to the similar behavior as discussed for the SF networks. What follows that multiplexing of random (SF and ER) networks with 1d lattice favors more to the cluster synchronizability as compared to multiplexing with the random networks.

In the following, we explore the reasons behind the impact of change in the density of connections in the second layer on the cluster synchronizability of first layer at strong couplings by using a simple case. The difference variable, of two nodes in the first layer at $\varepsilon=1$, can be written as,

$$
\begin{array}{r}
x_{i}^{1}(t+1)-x_{j}^{1}(t+1)=\frac{1}{k_{i}^{1}+1}\left(\sum_{j=1}^{N}\left(A_{i j}^{1} f\left(x_{i}^{1}(t)\right)\right)\right)- \\
\frac{1}{k_{j}^{1}+1}\left(A_{j i}^{1} f\left(x_{j}^{1}(t)\right)\right)+\left(\frac { 1 } { k _ { i } ^ { 1 } + 1 } f \left(x_{i}^{2}(t)-\right.\right. \\
\left.\left.\frac{1}{k_{j}^{1}+1} f\left(x_{j}^{2}(t)\right)\right)\right),
\end{array}
$$

where superscripts 1 and 2 stand for the first and second layer respectively. If global synchronization is achieved in the second layer, due to its denseness, in the above variable the coupling term having contribution from the second layer will get cancel out provided these pair of nodes have same degree $\left(k_{i}=k_{j}\right)$. Consequently the syn- 
chronization between two nodes will depend only on the properties of isolated network. For example, the sparse 1$\mathrm{d}$ lattice upon multiplexing with dense random networks exhibits no cluster synchronization as observed for the isolated network (Fig. 2(a)).

Furthermore, we study the change in the mechanism behind the cluster formation due to multiplexing. At weak couplings, the $\mathrm{D}$ synchronization remains the prime mechanism behind the cluster formation after multiplexing (Fig. [3(a)) as observed for the isolated networks [20], while at the intermediate and strong couplings the connection density of the second layer plays an important role. In this coupling regime, multiplexing with the sparse networks leads to a change in the mechanism of cluster synchronization(Fig. 3(b) and Fig. 4), whereas upon multiplexing with the dense networks though there is a suppression in cluster synchronization but the mechanism behind cluster formation remains same (Fig. 3(b)). In order to understand this impact of the multiplexing on the mechanism of the cluster formation, we investigate cluster synchronizability of a three nodes network. We find that the synchronization among the nodes in the same layer is suppressed due to an enhancement in the synchronization between the nodes of the different layers. What follows that the suppression in the SO synchronization at the strong couplings occurs due to the synchronization between nodes which are counter part of each other in different layers, whereas the D synchronization between a pair of node remains unaffected due to the same coupling environment they receive. As in Fig. 7(a), occurrence of synchronization between node 2 in first layer with its counter part in the second layer suppresses the synchronization between nodes 2, 3 and 2, 1 while the nodes 1 and 3 remain synchronized as these nodes still receive a common coupling from node 2 .

To conclude, we have studied the impact of multiplexing on the cluster synchronizability and mechanism behind the synchronization of a layer in the simplest multiplex network consisting of a network. We find that

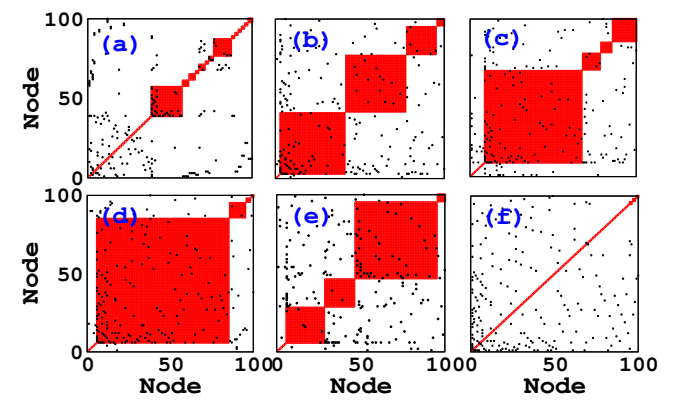

FIG. 6: Node versus node diagram (a) for the isolated SF network with $N=100$ and $\left\langle k_{2}\right\rangle=2$, (b), (c), (d), (e) and (f) after multiplexing with a layer represented by ER random network with $\left.k_{2}\right\rangle=4,6,8,10,16$ respectively at $\varepsilon=0.8$.

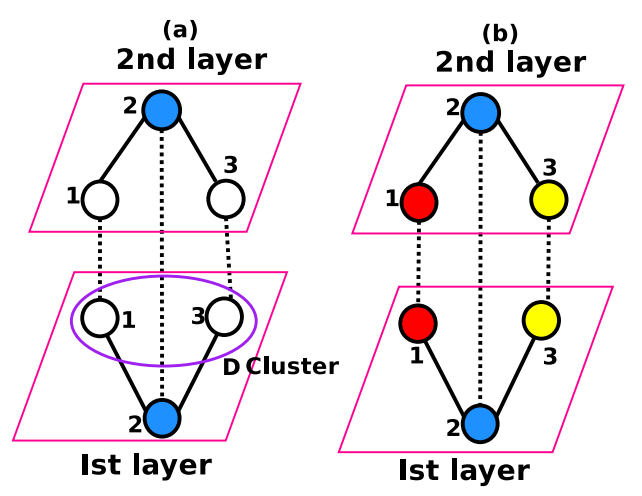

FIG. 7: Schematic diagrams depicting a multiplex network with three nodes in each layer. (a) formation of D cluster (nodes within the circle) when node 2 is synchronized with its counter part (denoted with same shade (color), (b) complete suppression in synchronization due to synchronization between the all the nodes (denoted with their counter parts (color)).

at weak couplings, the multiplexing enhances the cluster synchronizability, while at the strong couplings this enhancement depends on the architecture as well as the connection density of the another layer. The cluster synchronizability of a layer is enhanced when another layer has a moderate connection density. Moreover, multiplexing favors to the enhancement in the cluster synchronization when one of the layer is random and another is regular. The enhancement in the cluster synchronization is also associated with the change in the mechanism of the cluster formation. The multiplexing primarily influ- 
ences the synchronization between the nodes which are directly connected while leaving the synchronization between other pairs of nodes unaffected.

Our work demonstrates that in a multiplex network, the activity in a layer (sub-network) is significantly influenced by the structural properties of another layer (subnetwork). If connection density in one layer increases above a certain limit, it may spoil the synchronization in the another layer. The results presented here, about dynamical behavior of multiplex networks, may provide a guidance for the construction of a better model networks with multiplex architecture, such as the airport networks composing different airline companies [24].

SJ acknowledges CSIR (25(0205)/12/EMR-II) for the financial support. AS thanks complex systems lab members, specially Alok Yadav for useful discussions.
[1] A. Pikovsky, M. Rosenblum and J. Kurths, Synchronization: A Universal Concept in Nonlinear Sciences (Cambridge Univ. Press, Cambridge 2003); A. Arenas et. al., Phys. Rep. 469, 93 (2008).

[2] S. Boccaletti, The Synchronized Dynamics of Complex Systems (Elsevier Science Ltd., (2008)).

[3] A. R. Laird, B. P. Rogers and M. E. Meyerand, Proc. Intl. Soc. Mag. Reson. Med. 10 (2012); F. Varela et. al., Nat. Rev. Neuroscience 2, 229 (2001).

[4] A. Arenas et. al., Phys. Rep. 469, 93 (2008).

[5] S. Jalan and R. E. Amritkar, Phys. Rev. Lett. 90, 014101, (2003); S. Jalan, R. E. Amritkar and C.-K. Hu, Phys. Rev. E 72, 016211 (2005).

[6] B. Blasius, A. Huppert and L. Stone, Nature (London) 399, 354 (1999); A. Schnitzler and J. Gross, Nat. Rev. Neurosci. 6, 285 (2005); J. Kestler et. al., Phys. Rev. E 76, 035202 (2007); I. Kanter et. al., EPL 93, 66001 (2011); A. E. Motter et. al., Nature Phys. 9, 191 (2013); A. Palmigiano et. al., PloS One 7, 41799 (2012).

[7] M. R. Tinsley, S. Nkomo and K. Showalter, Nat. Phys. Lett. 8, 662 (2012).

[8] V. N. Belykh, I. V. Belykh and E. Mosekilde, Phys. Rev. E. 63, 036216 (2000).

[9] M. Nixon et. al., Phys. Rev. Lett. 106, 223901 (2011).

[10] T. Dahms, J. Lehnert and E. Schöll, Phys. Rev. E. 86, 016202 (2012).

[11] K. Wang, X. Fu and K. Li, Chaos 19, 023106 (2009).

[12] S. Boccaletti et al., Phys. Rep. (2014).

[13] M. Szella, R. Lambiotte and S. Thurner, Proc. Natl Acad. Sci. USA 107, 13636 (2010).
[14] M. Barthélemy, Phys. Rep. 499, 1 (2011).

[15] J. G. Gardenes et. al., Nature Scientific Reports 2, 620 (2012).

[16] C. Granell, S. Gomez, and A. Arenas, Phys. Rev. Lett. 111, 128701 (2013).

[17] J. Aguiree et. al., Phys. Rev. Lett. 112, 248701 (2014).

[18] A. Bogojeska et. al., Telecommunications Forum, TELFOR, 172 (2013).

[19] K. Kaneko, Phys. Rev. Lett. 63, 219 (1989).

[20] S. Jalan, R.E. Amritkar and C.-K. Hu, Phys. Rev. E 72, 016211 (2005).

[21] R. Albert and A.-L. Barabási, Rev. Mod. Phys. 74, 47 (2002).

[22] 1-D lattices used in the simulation have circular boundary conditions with each node having $k$ nearest neighbors. Small-world, scale-free and random networks are obtained by using SW, BA and ER model respectively 21. The complete bipartite networks consist of two sets where each node of one set is connected with all the nodes of other set. Results are presented for both the sets having equal number of nodes.

[23] Note that the definition of phase synchronization used here assign anti-phase synchronized nodes into two different clusters due to phase distance being one. However, this particular situation of nodes being anti-phase synchronized is not so often observed for chaotic evolution.

[24] A. Cardillo et al., Nature Scientific Reports 3, 1344 (2013). 